Supporting Information for

\title{
Does the Forest Filter Effect Prevent Semi-Volatile Organic Compounds from Reaching the Arctic?
}

Yushan Su and Frank Wania

\section{Extrapolating Dry Gaseous Deposition Velocities}

Since different forests have variable roughness and experience different local meteorological conditions, the dry gaseous deposition is likely to differ between forests. It is intented to estimate a dry gaseous deposition velocity for different forests from the gaseous deposition velocity $\left(\mathrm{V}_{\mathrm{D}}\right.$ G) measured in Bayreuth using information on roughness length and local wind speed, and a well established model of dry gaseous deposition. The "big-leaf dry deposition model" has been developed for modeling the dry gaseous deposition of inorganic chemicals to vegetation (Wesely, 1989). Good agreement between field measurements and modeling prediction was found for several inorganic chemicals (Brook et al. 1999 and Zhang et al. 2003). For example, $\mathrm{HNO}_{3}$ is one of the compounds well described by this model. In this model, the multiple resistance analogy approach is used (Eq. 1):

$$
\mathrm{V}_{\mathrm{D}-\mathrm{G}}=\frac{1}{\mathrm{R}_{\mathrm{A}}+\mathrm{R}_{\mathrm{B}}+\mathrm{R}_{\mathrm{C}}}=\frac{1}{\frac{\ln \left(\mathrm{Z} / \mathrm{Z}_{0}\right)-\psi}{\mathrm{k} \cdot \mathrm{U}^{*}}+\frac{5}{\mathrm{U}^{*}}\left(\frac{\mathrm{v}}{\mathrm{D}}\right)^{2 / 3}+\mathrm{R}_{\mathrm{C}}}
$$

$\mathrm{V}_{\mathrm{D}-\mathrm{G}} \quad$ dry gaseous deposition velocity

$\mathrm{R}_{\mathrm{A}} \quad$ bulk aerodynamic resistance

$\mathrm{R}_{\mathrm{B}} \quad$ quasi-laminar sublayer resistance

$\mathrm{R}_{\mathrm{C}}$ overall canopy resistance

$\mathrm{Z} \quad$ reference height at which the $\mathrm{V}_{\mathrm{D}-\mathrm{G}}$ is evaluated $\mathrm{Z}=30 \mathrm{~m}$ (typical value for forests)

$\mathrm{Z}_{0} \quad$ roughness length

$\Psi \quad$ stability correction parameter, in neutral or stable condition $\psi=0$

$\mathrm{K} \quad$ von Karman constant $\mathrm{k}=0.4$

U local wind speed

$\mathrm{U}^{*} \quad$ friction velocity

$$
\mathrm{U}^{*}=\mathrm{k} \cdot \mathrm{U} / \ln \left(\mathrm{Z} / \mathrm{Z}_{0}\right)
$$

D diffusivity of chemicals in air

$$
\mathrm{D}=4.0 \cdot 10^{-6} \mathrm{~m}^{2} / \mathrm{s}
$$


In neutral condition without considering diurnal variance, i.e. $\psi=0$, both $R_{A}$ and $R_{B}$ can be simplified to become a function of roughness length $Z_{0}$ and wind speed $U$ only:

$\mathrm{R}_{\mathrm{A}}=\left[\ln \left(\mathrm{Z} / \mathrm{Z}_{0}\right)\right]^{2} /\left(\mathrm{k}^{2} \cdot \mathrm{U}\right)=\mathrm{f}\left(\mathrm{Z}_{0}, \mathrm{U}\right)$

$\mathrm{R}_{\mathrm{B}}=\left[5 \cdot \ln \left(\mathrm{Z} / \mathrm{Z}_{0}\right) \cdot(\mathrm{v} / \mathrm{D})^{2 / 3}\right] /(\mathrm{k} \cdot \mathrm{U})=\mathrm{f}\left(\mathrm{Z}_{0}, \mathrm{U}\right)$

$\mathrm{R}_{\mathrm{C}}$ can be separated into two parallel components: stomatal resistance and non-stomatal resistance. The cuticle (non-stomatal part) plays an important role in trapping SOCs. Specifically, it is believed that for SOCs the stomatal resistance is much bigger than nonstomatal (cuticle) resistance. Because they are parallel, we may assume $\mathrm{R}_{\mathrm{C}} \approx \mathrm{R}_{\text {Cuticle. Thus: }}$

$\mathrm{V}_{\mathrm{D}-\mathrm{G}}=1 /\left(\mathrm{R}_{\mathrm{A}}+\mathrm{R}_{\mathrm{B}}+\mathrm{R}_{\text {Cuticle }}\right)$

As there appear to be no models available to derive $\mathrm{R}_{\text {Cuticle }}$, we suggest to treat $\mathrm{R}_{\text {Cuticle }}$ simply as a constant with different values for coniferous and deciduous forests.

\section{Gaseous Deposition to Deciduous Canopy}

In the N-Temperate zone, the measured maximum deposition velocity for SOCs to a deciduous forest canopy $\mathrm{V}_{\mathrm{D}-\mathrm{G}}$ is $3.6 \mathrm{~cm} / \mathrm{s}=0.036 \mathrm{~m} / \mathrm{s}$ (Horstmann and McLachlan, 1998). This dry gaseous deposition velocity is high and similar to those reported for $\mathrm{HNO}_{3}$ whose deposition is determined by the rate of transport from the atmosphere to canopy. It thus seems reasonable to assume that for SOC deposition to deciduous forests the sum $\left(R_{A}+R_{B}\right)$ is much larger than $\mathrm{R}_{\text {Cuticle }}$ - as is the case for $\mathrm{HNO}_{3}-$ and $\mathrm{R}_{\mathrm{C}}$ can be neglected.

The total resistance $\mathrm{R}$ thus is:

$\mathrm{R}=\mathrm{R}_{\mathrm{A}}+\mathrm{R}_{\mathrm{B}}=1 / \mathrm{V}_{\mathrm{D}-\mathrm{G}}=27.78(\mathrm{~s} / \mathrm{m})$ (deciduous forest in N-Temperate zone)

From Eq. 2 and Eq. 3, it is obvious that $\mathrm{R}_{\mathrm{A}}$ and $\mathrm{R}_{\mathrm{B}}$ are dependent on roughness length $\mathrm{Z}_{0}$ and very sensitive to local wind speed $U$. However, we do not know what $U$ and $Z_{0}$ was at the forest site in Bayreuth, Germany. We set $Z_{0}$ to $1.0 \mathrm{~m}$ based on a GIS-derived average value for deciduous forest in the Northern temperate zone, and then chose a value for the wind speed within the reasonable range ( 5 to $15 \mathrm{~m} / \mathrm{s}$ if deposition to forest canopy is evaluated at a height of $30 \mathrm{~m})$ such that $\mathrm{R}_{\mathrm{A}}+\mathrm{R}_{\mathrm{B}}$ equals $27.78(\mathrm{~s} / \mathrm{m})$. We further used the following parameter values: $\mathrm{Z}=$ $30 \mathrm{~m}, \mathrm{k}=0.4, v=1.55 \cdot 10^{-5} \mathrm{~m}^{2} / \mathrm{s}$, and $\mathrm{D}=4 \cdot 10^{-6} \mathrm{~m}^{2} / \mathrm{s}$.

Choosing a wind speed of $6.3 \mathrm{~m} / \mathrm{s}$ yields the appropriate sum of $\mathrm{R}_{\mathrm{A}}+\mathrm{R}_{\mathrm{B}}$ :

$\mathrm{R}_{\mathrm{A}}=[\ln (30 / 1.0)]^{2} /\left(0.4^{2} \cdot 6.3\right)=11.25 \mathrm{~s} / \mathrm{m}$

$\mathrm{R}_{\mathrm{B}}=5 \cdot \ln (30 / 1.0) \cdot\left(1.55 \cdot 10^{-5} / 4 \cdot 10^{-6}\right)^{2 / 3} /(0.4 \cdot 6.3)=16.49 \mathrm{~s} / \mathrm{m}$

$\mathrm{R}_{\mathrm{A}}+\mathrm{R}_{\mathrm{B}}=27.74 \mathrm{~s} / \mathrm{m}$

We assumed that $\mathrm{R}_{\text {Cuticle }}$ is always negligible for deciduous canopies, and then used equations 2 and 3 , as well as the spatially and monthly resolved GIS data for $Z_{0}$ and $U$, to calculate $\mathrm{V}_{\mathrm{D}-\mathrm{G} \text {. }}$

\section{Gaseous Deposition to Coniferous Canopy}

The measured deposition velocity to the coniferous forest in Bayreuth is $0.0078 \mathrm{~m} / \mathrm{s}$, thus

$\mathrm{R}=\mathrm{R}_{\mathrm{A}}+\mathrm{R}_{\mathrm{B}}+\mathrm{R}_{\text {Cuticle }}=1 / \mathrm{V}_{\mathrm{D}-\mathrm{G}}=1 / 0.0078=128.21(\mathrm{~s} / \mathrm{m})$ 
GIS-derived averaged roughness $Z_{0}$ for coniferous in N-Temperate is $1.1 \mathrm{~m}$. However, the monoculture spruce forest in Bayreuth presumably has a smaller roughness length, therefore, $\mathrm{Z}_{0}=0.5$ $\mathrm{m}$ is used here. All other parameters are the same as above, i.e. $\mathrm{U}=6.3 \mathrm{~m} / \mathrm{s}, \mathrm{Z}=30 \mathrm{~m}, \mathrm{k}=0.4, v$ $=1.55 \cdot 10^{-5} \mathrm{~m}^{2} / \mathrm{s}$, and $\mathrm{D}=4 \cdot 10^{-6} \mathrm{~m}^{2} / \mathrm{s}$.

$\mathrm{R}_{\mathrm{A}}=[\ln (30 / 0.5)]^{2} /\left(0.4^{2} \cdot 6.3\right)=15.37 \mathrm{~s} / \mathrm{m}$

$\mathrm{R}_{\mathrm{B}}=5 \cdot \ln (30 / 0.5) \cdot\left(1.55 \cdot 10^{-5} / 4 \cdot 10^{-6}\right)^{2 / 3} /(0.4 \cdot 6.3)=18.53 \mathrm{~s} / \mathrm{m}$

$\mathrm{R}_{\mathrm{A}}+\mathrm{R}_{\mathrm{B}}=33.91 \mathrm{~s} / \mathrm{m}$

Then, $\mathrm{R}_{\text {Cuticle }}=94.30 \mathrm{~s} / \mathrm{m}$

We suggest to assume that $\mathrm{R}_{\text {Cuticle }}$ has this value for all coniferous canopies in the world. $\mathrm{V}_{\mathrm{D}-\mathrm{G}}$ in other coniferous forest can thus be calculated by using equations 2 and 3, as well as the spatially and monthly resolved GIS data for $Z_{0}$ and U. All calculations regarding extrapolation of dry gaseous deposition velocities to different forests are listed in Table 5.

\section{Extrapolating Dry Particle-Bound Deposition Velocity}

A few well developed models seek to describe dry particle-bound deposition. However, most of these "process oriented" models rely heavily on knowledge of the particle size distribution of the chemicals of interest. Few publications have reported particle size distributions for SOCs, and most of them focused on PAHs and PCDD/Fs only. Therefore, we sought to avoid using such complicated models to extrapolate dry particle-bound deposition velocities. A simpler approach resembles the dry gaseous deposition model used above. Wesely et al. (1985) suggested such a "bulk resistance model" based on flux measurements of particulate $\mathrm{SO}_{4}{ }^{2-}$ over grassland. A dry particulate deposition velocity can be calculated as follows:

$\mathrm{V}_{\mathrm{D}-\mathrm{P}}=1 /\left(\mathrm{R}_{\mathrm{A}}+\mathrm{R}_{\mathrm{DS}}\right)$

In this particulate model, the resistance of the quasi-laminar sublayer $\left(R_{B}\right)$ and canopy $\left(R_{\text {Cuticle }}\right)$ is bulked together to give an overall resistance $\mathrm{R}_{\mathrm{DS}} . \mathrm{R}_{\mathrm{A}}$ is again the bulk aerodynamic resistance.

$\mathrm{R}_{\mathrm{A}}=\left[\ln \left(\mathrm{Z} / \mathrm{Z}_{0}\right)-\psi\right] /\left(\mathrm{k} \cdot \mathrm{U}^{*}\right)=\mathrm{f}\left(\mathrm{Z}_{0}, \mathrm{U}\right)$

A suggested formula for $\mathrm{R}_{\mathrm{DS}}$ for particulate $\mathrm{SO}_{4}{ }^{2-}$ deposition over grassland is

$\mathrm{R}_{\mathrm{DS}}=\mathrm{C} / \mathrm{U}^{*}$

where $\mathrm{U}^{*}$ is the friction velocity in $\mathrm{m} / \mathrm{s}$ and $\mathrm{C}$ is a constant $(\mathrm{C}=500)$. However, it is believed that the value of $\mathrm{C}$ in equation 5 is dependent on the vegetation type (i.e. here grass land) and it may not be applicable to coniferous and deciduous forests. We propose to use the measured particle deposition velocities to the two German forest canopies $V_{D-P}$ to deduce values of this constant $\mathrm{C}$ for deciduous and coniferous forests, respectively.

The measured dry particulate deposition velocity $V_{D-P}$ to deciduous forest is $0.73 \mathrm{~cm} / \mathrm{s}$, therefore:

$\mathrm{R}=1 / \mathrm{V}_{\mathrm{D}-\mathrm{G}}=137 \mathrm{~s} / \mathrm{m}$

$\mathrm{R}_{\mathrm{A}}=[\ln (30 / 1.0)]^{2} /\left(0.4^{2} \cdot 6.3\right)=11.25 \mathrm{~s} / \mathrm{m}$

$\mathrm{R}_{\mathrm{DS}}=\mathrm{R}-\mathrm{R}_{\mathrm{A}}=125.7 \mathrm{~s} / \mathrm{m}$

$\mathrm{C}_{\text {deciduous }}=\mathrm{U}^{*} / \mathrm{V}_{\mathrm{DS}}=\mathrm{U}^{*} \cdot \mathrm{R}_{\mathrm{DS}}=0.4 \cdot 6.3 / \ln (30 / 1.0) \cdot 125.7=94.1$ 
Similarly, the reported particulate deposition velocity for the coniferous canopy is $0.05 \mathrm{~cm} / \mathrm{s}$. The roughness length is set to 0.5 .

$\mathrm{R}=1 / \mathrm{V}_{\mathrm{D}-\mathrm{G}}=2000 \mathrm{~s} / \mathrm{m}$

$\mathrm{R}_{\mathrm{A}}=[\ln (30 / 0.5)]^{2} /\left(0.4^{2} \cdot 6.3\right)=15.37 \mathrm{~s} / \mathrm{m}$

$\mathrm{R}_{\mathrm{DS}}=\mathrm{R}-\mathrm{R}_{\mathrm{A}}=1984.5 \mathrm{~s} / \mathrm{m}$

$\mathrm{C}_{\text {coniferous }}=1321.4$.

We used equation 6 and these values $C$ to calculate $\mathrm{R}_{\mathrm{DS}}$ and further calculate dry particle-bound deposition velocities in the world's forests using equation 5. All calculations involved in deducting dry particulate deposition velocity are listed in Table 6.

\section{References}

Brook, J. R.; Zhang, L.; Di-Giovann; F., Padro, J. Description and evaluation of a model of deposition velocities for routine estimates of air pollutant dry deposition over North America. Part I: model development. Atmos. Environ. 1999, 33, 5037-5051.

Horstman, M.; McLachlan, M. S. Atmospheric deposition of semivolatile organic compounds to two forest canopies. Atmos. Environ. 1998, 32, 1799-1809.

Wesely, M. L.; Cook, D.R.; Hart, R. L.; Speer, R. E. Measurements and parameterization of particulate sulfur dry deposition over grass. J. Geophys. Res. [Atmospheres] 1985, 90(D1), 2131-2143.

Wesely M. L. Parameterization of surface resistances to gaseous dry deposition in regional-scale numerical models. Atmos. Environ. 1989, 23, 1293-1304.

Zhang, L.; Brook, J. R.; Vet, R. A revised parameterization for gaseous dry deposition in airquality models. Atmos. Chem. Phys., 2003, 3, 2067-2082. 
Table S1 Relative distribution of global forests and vegetation in the 10 climate zones of the Globo-POP model.

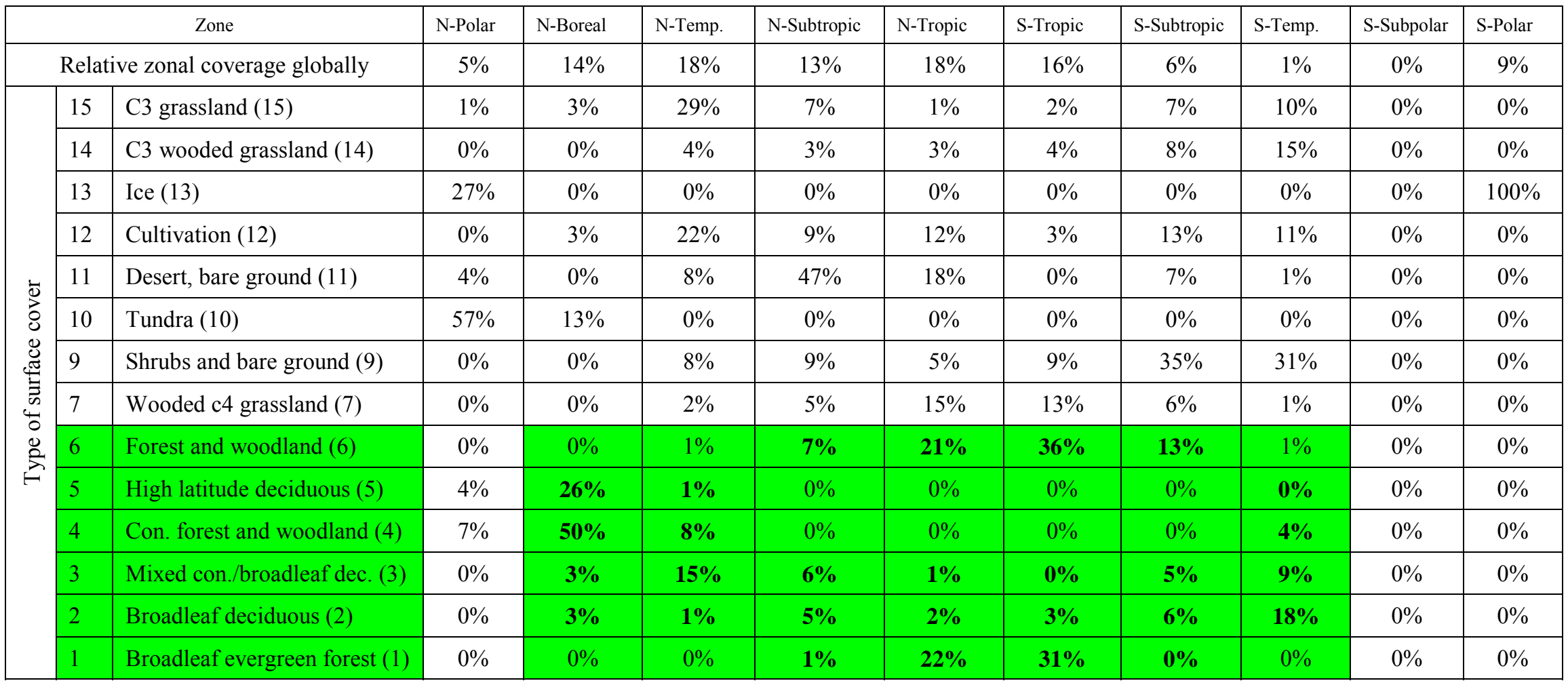

Table S2 Approach by which different forests types (vegetation type 1 to 6 in Table S1) were allocated to two forest compartments in each zone.

\begin{tabular}{|c|c|c|c|c|c|c|c|}
\hline & N-Tropic & S-Tropic & N-Subtropic & S-Subtropic & N-Temperate & S-Temperate & N-Boreal \\
\hline \multirow{2}{*}{$\begin{array}{l}\text { Forest } \\
\text { compartments }\end{array}$} & \multicolumn{2}{|c|}{$\begin{array}{l}\text { Evergreen forest } \\
(\# 1+\# 2+\# 3)\end{array}$} & \multicolumn{2}{|c|}{$\begin{array}{l}\text { High density deciduous } \\
(\# 2+\# 3)\end{array}$} & \multicolumn{2}{|l|}{$\begin{array}{l}\text { Coniferous } \\
\left(0.5^{*} \# 3+\# 4\right)\end{array}$} & $\begin{array}{l}\text { Coniferous } \\
\left(0.5^{*} \# 3+\# 4\right)\end{array}$ \\
\hline & \multicolumn{2}{|c|}{$\begin{array}{l}\text { Low density deciduous } \\
\text { (\#6) }\end{array}$} & \multicolumn{2}{|c|}{$\begin{array}{l}\text { Low density deciduous } \\
(\# 6)\end{array}$} & \multicolumn{2}{|c|}{$\begin{array}{l}\text { High density deciduos } \\
\left(0.5^{*} \# 3+\# 2+\# 5\right)\end{array}$} & $\begin{array}{l}\text { Low density deciduous } \\
\left(0.5^{*} \# 3+\# 2+\# 5\right)\end{array}$ \\
\hline
\end{tabular}


Table S3 Extrapolation of specific forest canopy volumes.

\begin{tabular}{|c|c|c|c|c|c|c|c|c|c|c|c|c|c|c|c|}
\hline \multirow{2}{*}{\multicolumn{2}{|c|}{\begin{tabular}{|c|} 
Zone \\
Forest type
\end{tabular}}} & \multicolumn{2}{|c|}{ N-Boreal } & \multicolumn{2}{|c|}{ N-Temperate } & \multicolumn{2}{|c|}{ N-Subtropic } & \multicolumn{2}{|c|}{ N-Tropic } & \multicolumn{2}{|c|}{ S-Tropic } & \multicolumn{2}{|c|}{ S-Subtropic } & \multicolumn{2}{|c|}{ S-Temperate } \\
\hline & & Con. & $\begin{array}{l}\text { Low } \\
\text { density } \\
\text { Dec. }\end{array}$ & Con. & $\begin{array}{l}\text { High } \\
\text { density } \\
\text { Dec. }\end{array}$ & $\begin{array}{l}\text { High } \\
\text { density } \\
\text { Dec. }\end{array}$ & \begin{tabular}{|c} 
Low \\
density \\
Dec.
\end{tabular} & $\begin{array}{c}\text { Evergreen } \\
\text { forest }\end{array}$ & $\begin{array}{l}\text { Low } \\
\text { density } \\
\text { Dec. }\end{array}$ & $\begin{array}{c}\text { Evergreen } \\
\text { forest }\end{array}$ & $\begin{array}{l}\text { Low } \\
\text { density } \\
\text { Dec. }\end{array}$ & $\begin{array}{l}\text { High } \\
\text { density } \\
\text { Dec. }\end{array}$ & $\begin{array}{l}\text { Low } \\
\text { density } \\
\text { Dec. }\end{array}$ & Con. & $\begin{array}{l}\text { High } \\
\text { density } \\
\text { Dec. }\end{array}$ \\
\hline \multirow{12}{*}{$\begin{array}{l}\text { GIS generated } \\
\text { LAI }\left(\mathrm{m}^{2} / \mathrm{m}^{2}\right)\end{array}$} & Jan. & 1.43 & 0.10 & 1.04 & 0.47 & 1.41 & 0.66 & 3.92 & 1.15 & 4.03 & 1.99 & 2.19 & 2.25 & 3.90 & 4.08 \\
\hline & Feb. & 1.40 & 0.12 & 0.90 & 0.39 & 1.27 & 0.58 & 3.61 & 0.83 & 3.74 & 2.03 & 2.19 & 2.33 & 3.84 & 3.40 \\
\hline & Mar. & 1.44 & 0.24 & 1.14 & 0.68 & 1.48 & 0.72 & 3.58 & 0.75 & 3.75 & 2.04 & 2.30 & 2.35 & 3.83 & 3.21 \\
\hline & Apr. & 1.53 & 0.63 & 1.70 & 1.32 & 1.90 & 1.05 & 3.57 & 0.86 & 3.77 & 2.03 & 2.44 & 2.16 & 3.60 & 3.12 \\
\hline & May & 2.05 & 1.61 & 2.72 & 2.41 & 2.39 & 1.48 & 3.59 & 1.14 & 3.79 & 1.93 & 2.30 & 1.69 & 2.56 & 2.31 \\
\hline & Jun. & 3.52 & 3.60 & 4.10 & 3.84 & 2.73 & 1.79 & 3.62 & 1.44 & 3.76 & 1.61 & 1.86 & 1.21 & 2.00 & 1.72 \\
\hline & Jul. & 4.25 & 5.05 & 4.57 & 4.26 & 2.79 & 1.87 & 3.66 & 1.71 & 3.72 & 1.18 & 1.63 & 0.95 & 1.87 & 1.47 \\
\hline & Aug. & 4.45 & 5.38 & 4.59 & 4.27 & 2.67 & 1.75 & 3.70 & 1.87 & 3.68 & 0.82 & 1.71 & 0.98 & 2.42 & 2.37 \\
\hline & Sept. & 4.37 & 5.33 & 4.36 & 4.04 & 2.69 & 1.65 & 3.72 & 1.96 & 3.64 & 0.67 & 2.02 & 1.23 & 3.25 & 3.77 \\
\hline & Oct. & 3.41 & 3.76 & 3.64 & 3.25 & 2.79 & 1.47 & 3.74 & 2.01 & 3.65 & 0.81 & 2.34 & 1.58 & 4.30 & 5.47 \\
\hline & Nov. & 1.70 & 1.29 & 2.22 & 1.99 & 2.58 & 1.23 & 3.73 & 1.79 & 3.67 & 1.18 & 2.27 & 1.71 & 4.57 & 5.55 \\
\hline & Dec. & 1.43 & 0.20 & 1.38 & 0.92 & 1.89 & 0.90 & 3.66 & 1.39 & 3.70 & 1.63 & 2.00 & 1.84 & 4.04 & 4.41 \\
\hline \multicolumn{2}{|c|}{$\operatorname{Max} \operatorname{LAI}\left(\mathrm{m}^{2} / \mathrm{m}^{2}\right)$} & & & 4.59 & 4.27 & & & & & & & & & & \\
\hline \multicolumn{2}{|c|}{ Thickness of leaf (m) } & 0.00037 & 0.00028 & 0.00037 & 0.00028 & 0.00028 & 0.00028 & 0.00028 & 0.00028 & 0.00028 & 0.00028 & 0.00028 & 0.00028 & 0.00037 & 0.00028 \\
\hline \multirow{12}{*}{$\begin{array}{c}\text { Specific } \\
\text { canopy } \\
\text { volume } \\
\left(\mathrm{m}^{3} / \mathrm{m}^{2}\right)\end{array}$} & Jan. & 0.00053 & 0.00003 & 0.00039 & 0.00013 & 0.00040 & 0.00018 & 0.00110 & 0.00032 & 0.00113 & 0.00056 & 0.00061 & 0.00063 & 0.00145 & 0.00115 \\
\hline & Feb. & 0.00052 & 0.00003 & 0.00033 & 0.00011 & 0.00036 & 0.00016 & 0.00101 & 0.00023 & 0.00105 & 0.00057 & 0.00061 & 0.00065 & 0.00142 & 0.00096 \\
\hline & Mar. & 0.00053 & 0.00007 & 0.00042 & 0.00019 & 0.00041 & 0.00020 & 0.00100 & 0.00021 & 0.00105 & 0.00057 & 0.00064 & 0.00066 & 0.00142 & 0.00090 \\
\hline & Apr. & 0.00057 & 0.00018 & 0.00063 & 0.00037 & 0.00053 & 0.00030 & 0.00100 & 0.00024 & 0.00106 & 0.00057 & 0.00069 & 0.00061 & 0.00133 & 0.00088 \\
\hline & May & 0.00076 & 0.00045 & 0.00101 & 0.00068 & 0.00067 & 0.00042 & 0.00101 & 0.00032 & 0.00106 & 0.00054 & 0.00065 & 0.00047 & 0.00095 & 0.00065 \\
\hline & Jun. & 0.00131 & 0.00101 & 0.00152 & 0.00108 & 0.00077 & 0.00050 & 0.00102 & 0.00040 & 0.00106 & 0.00045 & 0.00052 & 0.00034 & 0.00074 & 0.00048 \\
\hline & Jul. & 0.00158 & 0.00142 & 0.00169 & 0.00120 & 0.00078 & 0.00052 & 0.00103 & 0.00048 & 0.00105 & 0.00033 & 0.00046 & 0.00027 & 0.00069 & 0.00041 \\
\hline & Aug. & 0.00165 & 0.00151 & 0.00170 & 0.00120 & 0.00075 & 0.00049 & 0.00104 & 0.00053 & 0.00103 & 0.00023 & 0.00048 & 0.00028 & 0.00090 & 0.00067 \\
\hline & Sept. & 0.00162 & 0.00150 & 0.00162 & 0.00113 & 0.00075 & 0.00046 & 0.00104 & 0.00055 & 0.00102 & 0.00019 & 0.00057 & 0.00035 & 0.00120 & 0.00106 \\
\hline & Oct. & 0.00127 & 0.00106 & 0.00135 & 0.00091 & 0.00078 & 0.00041 & 0.00105 & 0.00057 & 0.00102 & 0.00023 & 0.00066 & 0.00044 & 0.00159 & 0.00154 \\
\hline & Nov. & 0.00063 & 0.00036 & 0.00082 & 0.00056 & 0.00072 & 0.00035 & 0.00105 & 0.00050 & 0.00103 & 0.00033 & 0.00064 & 0.00048 & 0.00170 & 0.00156 \\
\hline & Dec. & 0.00053 & 0.00006 & 0.00051 & 0.00026 & 0.00053 & 0.00025 & 0.00103 & 0.00039 & 0.00104 & 0.00046 & 0.00056 & 0.00052 & 0.00150 & 0.00124 \\
\hline
\end{tabular}


Table S4 Extrapolation of dry gaseous deposition velocities to forest.

\begin{tabular}{|c|c|c|c|c|c|c|c|c|c|c|c|c|c|c|}
\hline & \multicolumn{2}{|c|}{ N-Boreal } & \multicolumn{2}{|c|}{ N-Temperate } & \multicolumn{2}{|c|}{ N-Subtropic } & \multicolumn{2}{|c|}{ N-Tropic } & \multicolumn{2}{|c|}{ S-Tropic } & \multicolumn{2}{|c|}{ S-Subtropic } & \multicolumn{2}{|c|}{ S-Temperate } \\
\hline & Con. & $\begin{array}{l}\text { Low } \\
\text { density } \\
\text { Dec. }\end{array}$ & Con. & $\begin{array}{l}\text { High } \\
\text { density } \\
\text { Dec. }\end{array}$ & \begin{tabular}{|c|} 
High \\
density \\
Dec.
\end{tabular} & $\begin{array}{l}\text { Low } \\
\text { density } \\
\text { Dec. }\end{array}$ & $\begin{array}{c}\text { Evergreen } \\
\text { forest }\end{array}$ & $\begin{array}{l}\text { Low } \\
\text { density } \\
\text { Dec. }\end{array}$ & $\begin{array}{c}\text { Evergreen } \\
\text { forest }\end{array}$ & $\begin{array}{l}\text { Low } \\
\text { density } \\
\text { Dec. }\end{array}$ & $\begin{array}{l}\text { High } \\
\text { density } \\
\text { Dec. }\end{array}$ & $\begin{array}{l}\text { Low } \\
\text { density } \\
\text { Dec. }\end{array}$ & Con. & $\begin{array}{c}\text { High } \\
\text { density } \\
\text { Dec. }\end{array}$ \\
\hline Temperature in ${ }^{\circ} \mathrm{C}$ & 14 & 14 & 20 & 20 & 27 & 27 & 27 & 27 & 25 & 25 & 25 & 25 & 15 & 15 \\
\hline Temperature in $\mathrm{K}$ & 287.2 & 287.2 & 293.2 & 293.2 & 300.2 & 300.2 & 300.2 & 300.2 & 298.2 & 298.2 & 298.2 & 298.2 & 288.2 & 288.2 \\
\hline $\begin{array}{l}\text { Kinematic viscosity of air } \\
\left({ }^{*} 10^{5} \mathrm{~m}^{2} / \mathrm{s}\right)\end{array}$ & 1.50 & 1.50 & 1.55 & 1.55 & 1.62 & 1.62 & 1.62 & 1.62 & 1.60 & 1.60 & 1.60 & 1.60 & 1.51 & 1.51 \\
\hline Roughness $\mathrm{Z}_{0}(\mathrm{~m})$ & 1.2 & 1.2 & 0.5 & 1.0 & 1.0 & 0.1 & 2.6 & 0.1 & 2.6 & 0.1 & 0.8 & 0.1 & 1.1 & 1.1 \\
\hline Normalized wind speed & 1.06 & 1.28 & 1.19 & 1.1 & 1.37 & 1.54 & 0.7 & 0.63 & 0.67 & 1.04 & 1.59 & 1.54 & 0.74 & 0.49 \\
\hline Wind speed $(\mathrm{m} / \mathrm{s})$ & 6.07 & 7.33 & 6.8 & 6.30 & 7.85 & 8.82 & 4.01 & 3.61 & 3.84 & 5.96 & 9.11 & 8.82 & 4.24 & 2.81 \\
\hline Friction velocity $U^{*}(\mathrm{~m} / \mathrm{s})$ & 0.75 & 0.91 & 0.67 & 0.75 & 0.92 & 0.65 & 0.66 & 0.26 & 0.63 & 0.44 & 1.02 & 0.65 & 0.52 & 0.34 \\
\hline $\ln \left(\mathrm{Z} / \mathrm{Z}_{0}\right)$ & 3.23 & 3.22 & 4.09 & 3.37 & 3.42 & 5.45 & 2.45 & 5.45 & 2.43 & 5.45 & 3.57 & 5.45 & 3.27 & 3.31 \\
\hline $\mathrm{R}_{\mathrm{A}}(\mathrm{s} / \mathrm{m})$ & 10.77 & 8.86 & 15.37 & 11.25 & 9.32 & 21.05 & 9.33 & 51.51 & 9.64 & 31.20 & 8.72 & 21.06 & 15.75 & 24.46 \\
\hline $\mathrm{R}_{\mathrm{B}}(\mathrm{s} / \mathrm{m})$ & 16.05 & 13.25 & 18.53 & 16.49 & 13.81 & 19.59 & 19.34 & 47.90 & 19.94 & 28.80 & 12.31 & 19.44 & 23.33 & 35.72 \\
\hline $\mathrm{R}_{\mathrm{A}}+\mathrm{R}_{\mathrm{B}}(\mathrm{s} / \mathrm{m})$ & 26.82 & 22.11 & 33.91 & 27.75 & 23.13 & 40.64 & 28.68 & 99.41 & 29.59 & 60.00 & 21.04 & 40.50 & 39.08 & 60.18 \\
\hline $\mathrm{R}_{\mathrm{C}}(\mathrm{s} / \mathrm{m})$ & 94.30 & 0.03 & 94.30 & 0.03 & 0.03 & 0.03 & 0.03 & 0.03 & 0.03 & 0.03 & 0.03 & 0.03 & 94.30 & 0.03 \\
\hline $\mathrm{R}(\mathrm{s} / \mathrm{m})$ & 121.12 & 22.14 & 128.21 & 27.78 & 23.16 & 40.67 & 28.71 & 99.44 & 29.61 & 60.02 & 21.07 & 40.53 & 133.38 & 60.21 \\
\hline Calculated V $(\mathrm{cm} / \mathrm{s})$ & 0.83 & 4.52 & 0.78 & 3.60 & 4.32 & 2.46 & 3.48 & 1.01 & 3.38 & 1.67 & 4.75 & 2.47 & 0.75 & 1.66 \\
\hline
\end{tabular}


Table S5 Extrapolation of dry particle-bound deposition velocities to forests.

\begin{tabular}{|c|c|c|c|c|c|c|c|c|c|c|c|c|c|c|}
\hline & \multicolumn{2}{|c|}{ N-Boreal } & \multicolumn{2}{|c|}{ N-Temperate } & \multicolumn{2}{|c|}{ N-Subtropic } & \multicolumn{2}{|c|}{ N-Tropic } & \multicolumn{2}{|c|}{ S-Tropic } & \multicolumn{2}{|c|}{ S-Subtropic } & \multicolumn{2}{|c|}{ S-Temperate } \\
\hline & Con. & $\begin{array}{l}\text { Low } \\
\text { density } \\
\text { Dec. }\end{array}$ & Con. & $\begin{array}{c}\text { High } \\
\text { density } \\
\text { Dec. }\end{array}$ & $\begin{array}{c}\text { High } \\
\text { density } \\
\text { Dec. }\end{array}$ & $\begin{array}{c}\text { Low } \\
\text { density } \\
\text { Dec. }\end{array}$ & $\begin{array}{c}\text { Evergreen } \\
\text { forest }\end{array}$ & $\begin{array}{c}\text { Low } \\
\text { density } \\
\text { Dec. }\end{array}$ & $\begin{array}{c}\text { Evergreen } \\
\text { forest }\end{array}$ & $\begin{array}{c}\text { Low } \\
\text { density } \\
\text { Dec. }\end{array}$ & $\begin{array}{c}\text { High } \\
\text { density } \\
\text { Dec. }\end{array}$ & $\begin{array}{l}\text { Low } \\
\text { density } \\
\text { Dec. }\end{array}$ & Con. & $\begin{array}{l}\text { High } \\
\text { density } \\
\text { Dec. }\end{array}$ \\
\hline Roughness $Z_{0}(m)$ & 1.2 & 1.2 & 1.1 & 1.0 & 1.0 & 0.1 & 2.6 & 0.1 & 2.6 & 0.1 & 0.8 & 0.1 & 1.1 & 1.1 \\
\hline $\ln \left(Z / Z_{0}\right)$ & 3.23 & 3.22 & 3.27 & 3.37 & 3.42 & 5.45 & 2.45 & 5.45 & 2.43 & 5.45 & 3.57 & 5.45 & 3.27 & 3.31 \\
\hline Normalized wind speed & 1.06 & 1.28 & 1.19 & 1.10 & 1.37 & 1.54 & 0.70 & 0.63 & 0.67 & 1.04 & 1.59 & 1.54 & 0.74 & 0.49 \\
\hline Wind speed $(\mathrm{m} / \mathrm{s})$ & 6.1 & 7.3 & 6.8 & 6.3 & 7.8 & 8.8 & 4.0 & 3.6 & 3.8 & 6.0 & 9.1 & 8.8 & 4.2 & 2.8 \\
\hline Friction velocity $\mathrm{U}^{*}(\mathrm{~m} / \mathrm{s})$ & 0.75 & 0.91 & 0.83 & 0.75 & 0.92 & 0.65 & 0.66 & 0.26 & 0.63 & 0.44 & 1.02 & 0.65 & 0.52 & 0.34 \\
\hline $\mathrm{R}_{\mathrm{A}}(\mathrm{s} / \mathrm{m})$ & 10.77 & 8.86 & 9.79 & 11.25 & 9.32 & 21.05 & 9.33 & 51.51 & 9.64 & 31.20 & 8.72 & 21.06 & 15.75 & 24.46 \\
\hline $\begin{array}{l}\text { Constant for calculating } \\
V_{D S}\end{array}$ & 1660.6 & 94.1 & 1660.6 & 94.1 & 94.1 & 94.1 & 94.1 & 94.1 & 94.1 & 94.1 & 94.1 & 94.1 & 1660.6 & 94.1 \\
\hline $\mathrm{V}_{\mathrm{DS}}(\mathrm{m} / \mathrm{s})$ & 0.0005 & 0.0097 & 0.0005 & 0.0080 & 0.0098 & 0.0069 & 0.0070 & 0.0028 & 0.0067 & 0.0046 & 0.0109 & 0.0069 & 0.0003 & 0.0036 \\
\hline $\mathrm{R}_{\mathrm{DS}}(\mathrm{s} / \mathrm{m})$ & 2211.9 & 103.4 & 1990.2 & 125.7 & 102.5 & 145.3 & 143.5 & 355.4 & 149.1 & 215.3 & 92.1 & 145.4 & 3201.3 & 277.7 \\
\hline $\mathrm{R}(\mathrm{s} / \mathrm{m})$ & 2222.7 & 112.3 & 2000.0 & 137.0 & 111.8 & 166.4 & 152.9 & 406.9 & 158.8 & 246.5 & 100.8 & 166.4 & 3217.1 & 302.2 \\
\hline $\mathrm{V}(\mathrm{m} / \mathrm{s})$ & 0.0004 & 0.0089 & 0.0005 & 0.0073 & 0.0089 & 0.0060 & 0.0065 & 0.0025 & 0.0063 & 0.0041 & 0.0099 & 0.0060 & 0.0003 & 0.0033 \\
\hline Calculated V $(\mathrm{cm} / \mathrm{s})$ & 0.056 & 0.89 & 0.05 & 0.73 & 0.89 & 0.60 & 0.65 & 0.25 & 0.63 & 0.41 & 0.99 & 0.60 & 0.039 & 0.33 \\
\hline
\end{tabular}


Table S6 Transfer fluxes of three PCB congeners between N-Temperate and N-Boreal with and without forests (in \% of cumulative emissions from 1930 to 2000).

\begin{tabular}{|l|c|c|c|c|c|c|}
\hline \multirow{2}{*}{} & \multicolumn{3}{|c|}{ With forests } & \multicolumn{3}{c|}{ Without forests } \\
\cline { 2 - 7 } & PCB-52 & PCB-101 & PCB-180 & PCB-52 & PCB-101 & PCB-180 \\
\hline Transfer from N- Temperate to Boreal & 15.9 & 18.0 & 10.2 & 18.2 & 23.2 & 14.1 \\
\hline Transfer from N- Boreal to Temperate & 9.7 & 10.7 & 5.0 & 13.9 & 18.5 & 8.5 \\
\hline Net transfer from N- Temperate to Boreal & 6.2 & 7.3 & 5.2 & 4.3 & 4.7 & 5.6 \\
\hline
\end{tabular}

